\title{
The Manchester Microlensing Conference: the 12th International Conference and ANGLES Microlensing Workshop
}

\section{Eamonn Kerins}

Jodrell Bank Centre for Astrophysics, The University of Manchester, UK E-mail: Eamonn.Kerins@manchester.ac.uk

\section{Shude Mao}

Jodrell Bank Centre for Astrophysics, The University of Manchester, UK E-mail: Shude.Mao@manchester.ac.uk

\section{Nicholas Rattenbury}

Jodrell Bank Centre for Astrophysics, The University of Manchester, UK E-mail: Nicholas.Rattenbury@manchester.ac.uk

\section{Lukasz Wyrzykowski}

Institute of Astronomy, The University of Cambridge, UK

E-mail: wyrzykow@ast.cam.ac.uk

The Manchester Microlensing Conference:

The 12th International Conference and ANGLES Microlensing Workshop, January 21-25 2008, Manchester, UK 


\section{Preface}

The Manchester Microlensing Conference (M2C) was held at the Jodrell Bank Centre for Astrophysics at the University of Manchester in the UK from 21st-25th January 2008.

M2C comprised two elements: the ANGLES Microlensing Workshop and the 12th International Conference on gravitational microlensing. M2C began with the two-day Workshop, providing interactive Master Classes to around 60 researchers on selected hot topics in microlensing. The Master Classes were delivered by world-leading experts on each of the topics. The topics reflected the diverse techniques and applications of microlensing, such as crowded-field photometry, modelling of extra-solar planetary systems, and the use of microlensing in cosmology.

The 12th International Conference on microlensing followed immediately after the Workshop and was attended by around 90 researchers. The Conference covered all aspects of current research in microlensing, including: Microlensing towards the Magellanic Clouds; Cosmological Microlensing; Stellar and Galactic Microlensing; Galactic Microlensing Surveys; Follow-up Programmes and Planetary Microlensing; M31 Microlensing; and Future Directions.

The M2C Proceedings serve three functions. Through the expert master classes the M2C Proceedings provide a great starting point for those who wish to enter the field or who just wish to learn more about microlensing at a depth beyond that usually covered by a single review article. The $\mathrm{M} 2 \mathrm{C}$ proceedings also provide a snapshot of the state-of-the art in microlensing observations and theory as of January 2008, in what is a rapidly developing field. Lastly, the M2C meeting and its Proceedings are dedicated to the memory of the late Bohdan Paczyński, a towering figure and founding father of modern day microlensing research.

The Local Organizing Committee would like to thank several people for helping to ensure that the Workshop and Conference ran smoothly: Bob Dickson, Anthony Holloway and Chris Paul for flawless computing and AccessGrid support; Lisa McDermott for her tireless administrative support; Szymon Kozlowski for a wonderful poster design; and Edward Boyce, Dandan Xu, and Sarah Bryan for their invaluable assistance throughout the meeting. We would like to pay special thanks to Prof. Ian Browne for all of his help, advice and support. Finally, we are very grateful for the substantial financial support provided by the Astrophysics Network for Galaxy Lensing Studies (ANGLES), a European Union Sixth Framework research and training network.

7th February 2008

Eamonn Kerins

Shude Mao

Nicholas Rattenbury

Lukasz Wyrzykowski 

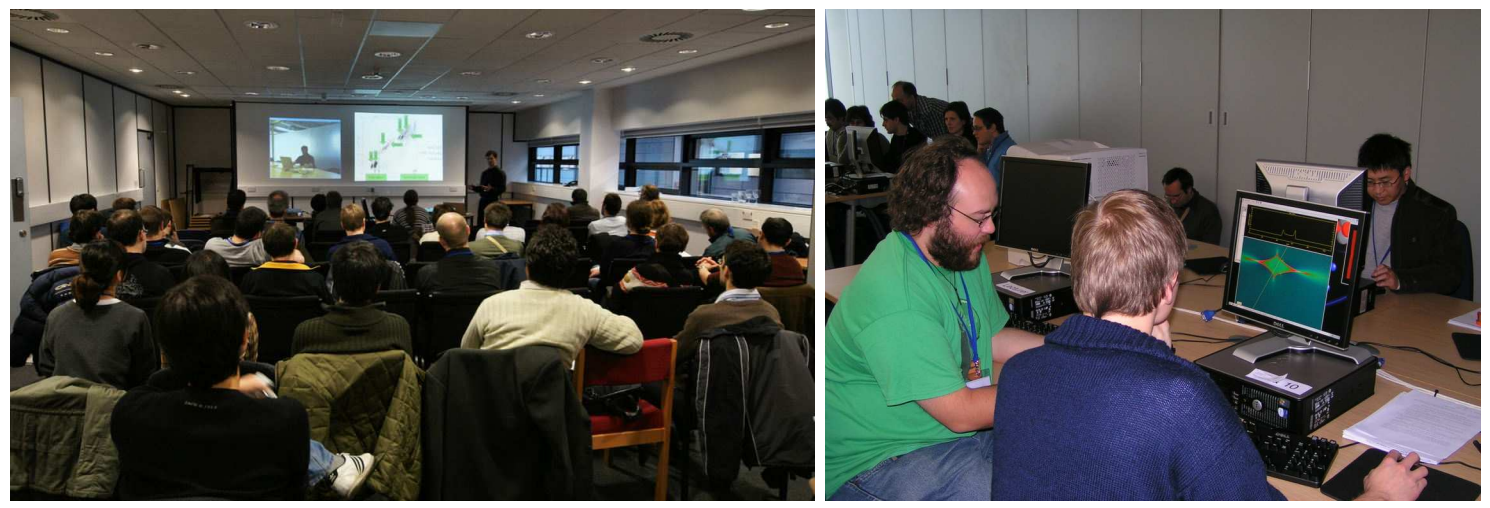

Figure 1: The ANGLES Workshop on microlensing. (Left) A master class partly delivered via a live video link through the University AccessGrid network. (Right) Workshop participants putting theory into practice by running a cosmological microlensing simulation (Photos: A. Holloway, L. Wyrzykowski).

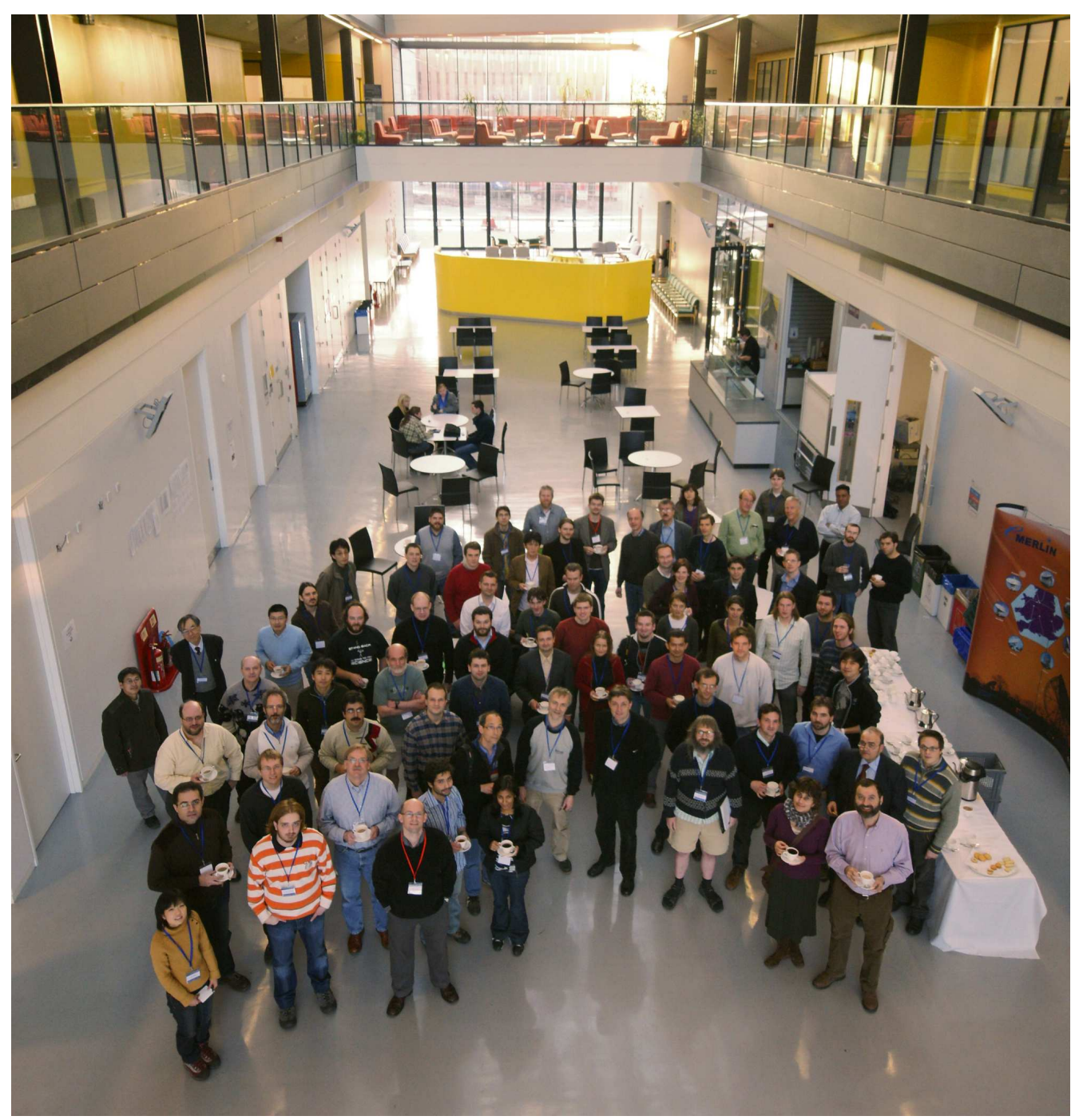

Figure 2: $\mathrm{M} 2 \mathrm{C}$ meeting participants enjoy a tea break in the atrium of the Alan Turing Building at the University of Manchester (Photo: A. Holloway). 


\section{List of Participants}

Timo Anguita

Jin An

Hideki Asada

Nicholas Bate

Virginie Batista

Jean-Philippe Beaulieu

Vasily Belokurov

David Bennett

Alexander Bergier

James Binney

Ian Bond

Valerio Bozza

Sebastiano Calchi Novati

Arnaud Cassan

Sun-ju Chung

Matt Darnley

Martin Dominik

Jadzia Donatowicz

Subo Dong

Jonathan Duke

Alexander Eigenbrod

Eva Eulaers

Wyn Evans

Janine Fohlmeister

Pascal Fouque

Cesar Fuentes

Arti Garg

Scott Gaudi

Andrew Gould

Cheongho Han

Frederic Hessman

David Heyrovsky

Tobias Cornelius Hinse

Shan Hongguang

Keith Horne

Markus Hundertmark

Richard Ignace

Christophe Jean

Philippe Jetzer

Noe Kains

Eamonn Kerins

Do-eon Kim

Szymon Kozlowski

Geraint Lewis

Luigi Mancini

Shude Mao
ARI / ZAH

NBIA, Copenhagen

Hirosaki University, Japan

The University of Melbourne

Institut d'Astrophysique de Paris

Institut d'Astrophysique de Paris

Institute of Astronomy

University of Notre Dame

Columbia University

Oxford University

Institute for Information and Mathematical Sciences

University of Salerno

Physics Department - University of Salerno

ARI, Heidelberg University

Chungbuk National Univ.

Liverpool John Moores University

SUPA, University of St Andrews

Technical Univeristy of Vienna, Austria

The Ohio State University

ARI (Liverpool John Moores Uni.) and ARI (Heidelberg)

Ecole Polytechnique Federale de Lausanne

Universite de Liege

Cambridge University

ARI Heidelberg

Observatoire Midi-Pyrenees

Harvard University

Harvard University

Ohio State University

Ohio State University

Chungbuk National University

Inst. f. Astrophysik, Univ. Goettingen

Institute of Theoretical Physics

Armagh Observatory

Yunnan Observatory, CAS

SUPA St.Andrews

Institut fuer Astrophysik, Univ. Goettingen

East Tennessee State University

Universidad de Valencia

Theoretical Physics University of Zurich

SUPA, University of St. Andrews

The University of Manchester

Chungbuk National University

Department of Astronomy, The Ohio State University

University of Sydney

Dipartimento di Fisica, Universit'a di Salerno

Jodrell Bank Centre for Astrophysics 
Jean-Baptiste Marquette

Dante Minniti

Marc Moniez

Yasushi Muraki

Petri Mutka

Maiko Nagaya

Seyed-Abouzar Najafi-Shoushatri

Andrew Newsam

Justin Nieusma

Byeong-Gon Park

Ondrej Pejcha

Sohrab Rahvar

Nicholas Rattenbury

Armin Rest

Davide Ricci

James Rich

Luca Santoro

Gaetano Scarpetta

Robert Schmidt

Jan Skowron

Dominique Sluse

Martin Smith

Colin Snodgrass

Denis Sullivan

Takahiro Sumi

Maciej Sznajder

Jonathan Thornburg

Yiannis Tsapras

Oleg Tsupko

Andrzej Udalski

Filomena Volino

Joachim Wambsganss

Thulsi Wickramasinghe

Przemek Wozniak

Lukasz Wyrzykowski

Philip Yock

Marta Zub
Institut d'Astrophysique de Paris

P. University Catolica de Chile

CNRS

Konan University

University of Oulu

Nagoya University

IPM, School of Astronomy and Astrophysics

Astrophysics Research Institute

The College of New Jersey

Korea Astronomy and Space Science Institute Institute of Theoretical Physics

Sharif Univ. of Tech.

Jodrell Bank Centre for Astrophysics

Harvard University

AEOS - University of Liege

Saclay

Dipartimento di Fisica, Universit'a di Salerno

Universit'a di Salerno

University of Heidelberg

Warsaw University Observatory

Ecole Polytechnique Federale de Lausanne (EPFL)

University of Cambridge

European Southern Observatory

Victoria University of Wellington, NZ

Nagoya University

Institute of Astronomy,University of Zielona Gora

University of Southampton

LCOGT

Space Research Institute of Russian Academy of Science

Warsaw University Observatory

Argelander-Institut fuer Astronomie

Heidelberg University (Zentrum fuer Astronomie)

The College of New Jersey

Los Alamos National Laboratory

Institute of Astronomy University of Cambridge

University of Auckland

ARI, Heidelberg University 